\title{
Adding to Relevant Restricted Quantification
}

\author{
JC BEALL* \\ UNIVERSITY OF CONNECTICUT, USA \\ UNIVERSITY OF OTAGO, NEW ZEALAND \\ http://entailments.net \\ jc. beall@uconn.edu \\ Received by Greg Restall \\ Published April I4, 2OII \\ http://www.philosophy.unimelb.edu.au/ajl/2011 \\ (C) $201 \mathrm{II}$ Jc Beall
}

\begin{abstract}
This paper presents, in a more general setting, a simple approach to 'relevant restricted generalizations' advanced in previous work. After reviewing some desiderata for restricted generalizations, I present the target route towards achieving the desiderata. An objection to the approach, due to David Ripley, is presented, followed by three brief replies, one from a dialetheic perspective and the others more general.
\end{abstract}

This paper presents, in a more general setting, a simple approach to 'relevant restricted generalizations' advanced in [2, Ch. 5]. The paper presupposes familiarity with standard issues of restricted generalizations in relevant-logic settings [7, 9], and in particular with the issues as framed in [3] with special attention on applications to robustly contraction-free theories $[\mathrm{I2}]$.$] Brief dis-$ cussion of target application-based issues is given in $\$$ I.2, but fuller and more leisurely discussion is confined to cited works.

*An objection to вхтт [2] by Vann McGee (in correspondence) occasioned the idea in this paper. The paper itself emerged in the very productive atmosphere of $\mathrm{WCP}_{4}$ (the $4^{\text {th }}$ World Congress of Paraconsistency), and I'm grateful to Graham Priest for discussion, and particularly grateful to Greg Restall, who noticed, over dinner, a simplification of the ideas I was thinking about. I'm also very grateful to Dave Ripley, who raised the objection discussed in this paper, and who continues to be a lively and valuable philosophical interlocutor, and to Aaron Cotnoir and Michael Hughes for useful discussion. Finally, were it not for the very interesting ideas that Richard S. Anderson has recently had on the topic, this paper would not be what it is.

IOne assumption throughout is that restricted generalizations involve a conditional. This is not an uncontroversial (or nonstandard) assumption. For other approaches, see Butchart $[5]$, Belnap [4], Slaney [13, I4] and references therein. I should note that issues concerning contraction, contraposition, and so on (see $8 \mathrm{I}$ ) emerge even if the current assumption about a conditional is rejected; the problems simply take a slightly different-conditional-free-form. 
The paper is structured as follows. \$I presents the desiderata advanced in [3], and $\$ \sqrt{2}$ presents the target route towards achieving the desiderata. An objection, due to David Ripley, is presented in $\$ 3 \$ 4$ offers three brief replies to the Ripley objection, one from a dialetheic perspective and the others more general. $\$ 5$ offers a few closing remarks.

\section{MINIMAL DESIDERATA}

Let $\mapsto$ be our target restricted conditional and $\rightarrow$ our given (detachable) relevant conditional $[2$ The desiderata, put forth in [3], are as follows (keeping to the given numbering) 3

\section{I.I GENERAL, A-LEVEL DESIDERATA}

Some general desiderata are as follows.

AI. $\alpha, \alpha \mapsto \beta \vdash \beta$ (Detachment).

A2. $\beta \vdash \alpha \mapsto \beta$ (Conditional Weakening).

A3. $\alpha \rightarrow \beta \vdash \alpha \mapsto \beta$.

A6. $\vdash \alpha \wedge \beta \mapsto \alpha$ (Conditional Simplification).

Note that [3] doesn't list A6, but it—like Conditional Identity (viz., $\vdash \alpha \mapsto \alpha)$ is a desideratum, at least if, as I presume, we want it to be logically true that (e.g.) all black cats are black and so on.

\section{I.2 APPLICATION-CENTERED, B-LEVEL DESIDERATA}

Some less general desiderata are as follows.

в г. $\alpha \mapsto \beta \nvdash \neg \beta \mapsto \neg \alpha$ (No Contraposition).

B2. $\alpha \mapsto(\alpha \mapsto \beta) \nvdash \alpha \mapsto \beta$ (No Contraction).

В2! More generally: robustly contraction-free in Restall's sense [12].

These desiderata emerge from target applications of the given logics, and in particular target 'semantic' theories as in [IO] and [2],4 Such theories have the resources to form Curry sentences, and so-given other logical features of the theories - the theories must remain 'robustly contraction-free' on pain of triviality. This motivates B2. Desideratum BI, in turn, is driven by target

${ }^{2}$ The assumption of detachability, made throughout, is rule detachability: $\alpha, \alpha \rightarrow \beta \vdash \beta$.

${ }^{3}$ I focus only on the $\mathrm{A}^{-}$and $\mathrm{B}-\mathrm{level}$ desiderata listed in [3] that involve the target conditional. (Also: I herein skip the c-level desiderata, all of which - I think (though have not proved)—are achievable on this approach (given suitable choices of quantifiers).)

${ }^{4}$ And see $[6$. In roughly the same family, though not paraconsistent, is Field's recent transparent truth theory $[8]$. 
'glutty' theories: theories containing $\alpha$ and $\neg \alpha$ for some $\alpha$. In such theories we cannot non-trivially add a conditional $\Rightarrow$ that satisfies what might be called the evil trinity:

- $\alpha, \alpha \Rightarrow \beta \vdash \beta$ (Detachment).

- $\beta \vdash \alpha \Rightarrow \beta$ (Conditional Weakening).

- $\alpha \Rightarrow \beta \vdash \neg \beta \Rightarrow \neg \alpha$ (Contraposition).

Suppose that we add $\Rightarrow$ to a glutty (and otherwise non-trivial) theory in the ballpark (e.g., B-ish theories), and let $\beta$ be a glut. By Conditional Weakening, $\beta$ gives us $\alpha \Rightarrow \beta$. By Contraposition, we have $\neg \beta \Rightarrow \neg \alpha$. But $\beta$ is a glut, and so we have $\neg \beta$, which, in concert with $\neg \beta \Rightarrow \neg \alpha$, yields $\neg \alpha$ via Detachment. And this is for all $\alpha$, and so we have near-enough triviality: $\neg \alpha$ for all $\alpha$. (If, as many of the target theories enjoy, we have $\neg \neg \alpha \vdash \alpha$, then we have triviality full stop.)

Of course, if one's application(s) of a given relevant logic are different from dialetheic or, more generally, robustly-contraction-free-demanding applications, then the B-level desiderata mightn't be pressing. Still, the general A-level desiderata are likely of interest.

\section{PROPOSAL: SIMPLE ADDITION}

Various proposals have been made towards achieving the given desiderata. For example, in [3], the Routley-Meyer ternary semantics (or simplified versions thereof) for one's given relevant conditional is assumed, and a new conditional is (semantically) defined via restrictions on the broader ternary relation. In [2, Ch. 5], an unsatisfiable sentence $\perp$ is invoked to define a candidate restricted conditional (among other discussed options). More famously, the Ackermann constant $\mathrm{t}$, axiomatized via $\alpha \dashv t \mathrm{t} \rightarrow \alpha$, is invoked to define a candidate restricted conditional (viz., $\alpha \wedge t \rightarrow \beta$ ) 5 And there are other proposals.

I leave the details, virtues and potential vices of such proposals to debate. Moreover, whether such proposals are needed in addition to the following proposal I also leave for debate. What is notable-and my chief aim here to noteis that, at least in many target logics, one needn't introduce further machinery to achieve the A-level (or, if one wants, the B-level) desiderata. One already has a candidate restricted conditional. In particular, where $\vee$ is disjunction, define

$$
\alpha \mapsto \beta:=(\alpha \rightarrow \beta) \vee \beta
$$

Assuming that disjunction and one's given relevant conditional (the arrow) behave properly, our restricted conditional, so defined, immediately delivers the given desiderata.

\footnotetext{
${ }^{5}$ Priest [IO endorses this sort of approach. For a non-triviality proof of adding the two-way axiom $\alpha \dashv \vdash \mathrm{t} \rightarrow \alpha$ to truth theory вхтт, see [2, Ch. 5].
} 


\section{I A-LEVEL DESIDERATA}

Details of particular logics are required for proofs, but 'semantic' considerations for B-vicinity logics point in the right direction.

- On AI: suppose that $\rightarrow$ Detaches and that $\alpha$ is true (in a suitable model). Then-assuming standard disjunction features—any model in which $\beta$ is untrue is one in which $(\alpha \rightarrow \beta) \vee \beta$ is untrue. So, $\mapsto$ itself Detaches.

- On A6: suppose that $\rightarrow$ Conditionally Simplifies, that is, that $\alpha \wedge \beta \rightarrow \alpha$ is true in all models. Then-assuming standard disjunction featuresevery model is one in which $(\alpha \wedge \beta \rightarrow \alpha) \vee \alpha$, and hence $\alpha \wedge \beta \mapsto \alpha$, is true in all models. So, $\mapsto$ itself Conditionally Simplifies.

Moreover, as is plain, we immediately get $\mathrm{A}_{2}$ and $\mathrm{A}_{3}$ if we have Addition (after which the 'simple addition' proposal is named): $\alpha \vdash \alpha \vee \beta$ and $\beta \vdash \alpha \vee \beta$.

- On A2: Addition gives us $\beta \vdash(\alpha \rightarrow \beta) \vee \beta$.

- On A3: Addition gives us $(\alpha \rightarrow \beta) \vdash(\alpha \rightarrow \beta) \vee \beta$.

\subsection{B-LEVEL DESIDERATA}

Whether we have the B-level desiderata turns on our given relevant conditional in terms of which our restricted conditional is defined. As in 2.1 genuine proofs require details of the particular logics or model theory, but rough, 'semantic' considerations point in the right direction.

- On вг: there are two cases, one in which the background conditional contraposes (as in BX) and one in which it doesn't.

Where $\rightarrow$ contraposes: take a model in which $\alpha \rightarrow \beta$ (and, hence, its contraposition) is untrue. Any such model in which $\beta$ is true but $\neg \alpha$ untrue is a countermodel to Contraposition for our restricted conditional; such a model is one in which $(\alpha \rightarrow \beta) \vee \beta$ is true but $(\neg \beta \rightarrow \neg \alpha) \vee \neg \alpha$ untrue. (There are such models in the B-vicinity contraction-free logics.)

Where $\rightarrow$ fails to contrapose: there are models in which $\alpha \rightarrow \beta$ is true but its contraposition untrue. Any such model in which $\neg \alpha$ is untrue is a countermodel to Contraposition for our restricted conditional; such a model is one in which $(\alpha \rightarrow \beta) \vee \beta$ is true but $(\neg \beta \rightarrow \neg \alpha) \vee \neg \alpha$ untrue. (There are such models in the B-vicinity contraction-free logics.)

- On в2: consider any model that invalidates Contraction for the background relevant conditional-that is, a model in which $\alpha \rightarrow(\alpha \rightarrow \beta)$ is true but $\alpha \rightarrow \beta$ untrue. Any such model in which $\beta$ is untrue is a countermodel to Contraction for our restricted conditional. So, в2 holds. 
- On в2!: whether we have robust freedom from contraction depends on the resources of the background logic (or target theory). Of course, if we have a non-triviality proof-as we do in some of the B-vicinity theorieswe thereby have a proof of robust freedom. ${ }^{6}$

What the foregoing indicates is that, given an appropriate background logic (and many of the relevant logics are appropriate in the target sense), we need not look further for an A-level restricted conditional (or B-level if we have it). We have such a conditional.

\section{RIPLEY OBJECTION}

According to David Ripley (correspondence), the foregoing proposal faces a problem. In short, there are models in which, intuitively, all As are Bs, but our restricted conditional is untrue in such models.

To make the point, we simplify by focusing on a standard, B-vicinity 'worlds' model in which all worlds are perfectly classical.7. For purposes of the example, we step up to the predicate-cum-quantifier level (with which level familiarity is assumed).8 Here, @ is the base world, in terms of which truth in a model is defined, and I is the interpretation function. The essential features of the model are as follows.

- Let $\mathcal{W}=\{@, w\}=\mathcal{N}$.

- Let the domain be $\{a, b, c\}$.

- Let $\mathrm{I}_{\Theta}(\alpha(x))=\{\mathrm{b}\}$ and $\mathrm{I}_{\Theta}(\beta(x))=\{\mathrm{a}, \mathrm{b}\}$.

- Let $\mathrm{I}_{w}(\alpha(x))=\{c\}$ and $\mathrm{I}_{w}(\beta(x))=\emptyset$.

The objection is that, intuitively, all As are Bs (at the actual world), since, at the actual world (viz., @), we have it that, for target sentences, $\mathrm{I}_{@}(\alpha(x)) \subseteq$ $\mathrm{I}_{@}(\beta(x))$. On the other hand, the generalization using the proposed restricted conditional, namely,

$$
\forall x(\alpha(x) \mapsto \beta(x))
$$

is not true at the actual world. In particular, the c-instance of $\alpha(x) \mapsto \beta(x)$ is untrue at@. After all, $\beta(\mathrm{c})$ is not true at @. Moreover, since there's a point $w$ at which $\alpha(\mathrm{c})$ is true but $\beta(\mathrm{c})$ untrue, $\alpha(\mathrm{c}) \rightarrow \beta(\mathrm{c})$ is untrue at @ too. Hence,

$$
(\alpha(c) \rightarrow \beta(c)) \vee \beta(c)
$$

\footnotetext{
${ }^{6}$ Moreover, such a proof would immediately establish freedom from the evil trinity of $\$$ I.2 Hence, e.g., in вхтт, where we have a non-triviality proof, we immediately know that since $\mapsto$ Detaches and satisfies Conditional Weakening, it thereby doesn't contrapose.

${ }^{7}$ Hence, as Ripley (in correspondence) notes, the given model is independent of paraconsistency issues: if it shows a problem with our proposed restricted conditional, it equally shows a problem with taking (say) the strict hook as a restricted conditional.

${ }^{8}$ For elementary discussion of these frameworks, see Beall [I], and for broader and more detailed (though equally user-friendly) discussion see Dunn \& Restall [7] or Priest [II].
} 
is untrue at @. Hence, assuming features of the quantifiers (which features are not in question here), the proposed restricted conditional fails to get the facts right —or so the objection goes.

\section{REMARKS ON THE RIPLEY OBJECTION}

The objection, I think, does not show a defect in the proposal. I give three replies: two very general (and only sketched), the other peculiar to target dialetheic theories (including вхтт, which I endorse). I begin with the latter.

\section{I A DIALETHEIC REPLY}

It is reasonable to expect that a candidate restricted conditional imply its hook counterpart. Indeed, this might well be made a desideratum-a D-level desideratum, we might say.

$$
\text { DI. } \alpha \mapsto \beta \vdash \alpha \supset \beta
$$

That our simple-addition conditional yields Di may be seen along standard 'semantic' lines-for example, simplified semantics for B-vicinity logics. Suppose that @ $\not \neq \alpha \supset \beta$, in which case @ $\models \alpha$ and @ $\not \mid \neg \alpha$ but @ $\not \neq \beta$. But, then, @ $\models(\alpha \rightarrow \beta) \vee \beta$ only if @ $\models \alpha \rightarrow \beta$, in which case, because $\rightarrow$ detaches, @ $\models \beta$. But this is impossible since $@ \not \models \beta$. Hence, there's no model in which $(\alpha \rightarrow \beta) \vee \beta$ is true and $\alpha \supset \beta$ not. Hence, by definition of our simple-addition conditional, there's no model in which $\alpha \mapsto \beta$ is true but $\alpha \supset \beta$ not.

On the other hand, the converse of DI, namely,

$$
\text { ID. } \alpha \supset \beta \vdash \alpha \mapsto \beta
$$

is dubious for various reasons, particularly in a dialetheic context. In standard dialetheic theories [2, IO], ID yields triviality if the restricted conditional $\mapsto$ detaches. (Proof: let $\alpha$ be a glut, in which case $\alpha \supset \beta$ is true, and so, via ID, $\alpha \mapsto \beta$ is true. But $\mapsto$ detaches, and so $\beta$ is true. Triviality.) So, ID is not an option for non-trivial dialetheic theories. Hence, in addition to DI, another D-level desideratum is failure of DI's converse:

D2. $\alpha \supset \beta \nvdash \alpha \mapsto \beta$

And this brings us back to Ripley's objection.

\section{I.I RIPLEY THE GOOD}

A positive way of seeing Ripley's 'objection' is not so much as an objection as a concrete confirmation that we have what we want: Ripley's model is confirmation of D2 (i.e., a countermodel to ID). We have @ $\models \forall x(A(x) \supset B(x))$ but @ $\forall \forall \forall x(A(x) \mapsto B(x))$. This is good. 


\section{I.2 RIPLEY THE BAD}

A negative way of seeing Ripley's objection is that it simply demands too much 9 The objection demands a constraint on the truth-at-a-point conditions (the 'truth conditions', if you want) of our restricted conditional. The demand amounts to one of the following constraints, where $\mathrm{I}_{w}(\mathrm{E})$ and $\mathrm{I}_{w}^{-1}(\mathrm{E})$ are, respectively, the extension and antiextension of expression $E$ at point $w$.

$$
\begin{aligned}
& \text { RI. } w \models \forall x(\alpha(x) \mapsto \beta(x)) \text { iff } \mathrm{I}_{w}(\alpha(x)) \subseteq \mathrm{I}_{w}(\beta(x)) \text {. } \\
& \text { R2. } w \models \forall x(\alpha(x) \mapsto \beta(x)) \text { iff } y \in \mathrm{I}_{w}^{-1}(\alpha(x)) \text { or } y \in \mathrm{I}_{w}(\beta(x)) \text { for any } y \text { in the }
\end{aligned}
$$
domain.

RI maintains that what's necessary and sufficient for the truth of a restricted generalization at a point $w$ is that the extension of $\alpha(x)$ at $w$ be a subset of the extension of $\beta(x)$ at $w$, while $\mathrm{R} 2$ invokes the condition that something's either in the antiextension of $\alpha(x)$ at $w$ or in the extension of $\beta(x)$ at $w$. Without at least one of RI and R2, the Ripley objection does not go through. The trouble, however, is that RI and R2 demand too much if, as I have assumed, subsets are understood along a classical (say, ZFC) theory. In short, either condition results in a contracting conditional, thereby undermining one of the main desiderata.

That a contracting conditional results from RI, where subsethood is understood along the assumed zFC lines, may be seen by noting that RI simply imposes the logical behaviour of our (classical) subset relation $\subseteq$ on the target restricted conditional. Since the former itself contracts (and detaches) so too does the latter. So, this demand is too much. (But see $\$ 4.2$ for further comment.)

That $\mathrm{R} 2$ is equally problematic may be seen by noticing that, at least for target logics, it requires our restricted conditional to have precisely the truthat-a-point conditions that the hook $\alpha \supset \beta$, defined as $\neg \alpha \vee \beta$, 'enjoys' ${ }^{10}$ But, then, this will fail to detach in target frameworks (or, in some, contract), and so fail at least some of the application-driven desiderata.

\subsection{A GENERAL REPLY}

A very brief but more general reply is available ${ }^{1}$ Ripley's objection charges that from $A \subseteq B$ we ought to have a true restricted generalization that all $A s$

\footnotetext{
9I am grateful to both David Ripley and, in particular, Richard S. Anderson for correspondence that led to a clearer formulation of this section.

${ }^{10}$ A Routley-Star approach to negation makes the conditions more involved, but the point goes through for such frameworks.

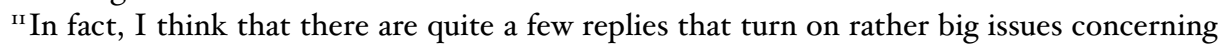
truth, meaning, model theory, and more. (E.g., if, as some deflationists might think, we are using the model theory as a mere heuristic guide to the logic, or at any rate not taking it to model 'real truth conditions', it is not obvious that the objection raises any problems for the logic of the proposed conditional.) The issues are both interesting and important, but more attention to them is demanded than I can give in this paper.
} 
are $\mathrm{Bs}$. In turn, the objection highlights cases in which the subset relation holds but in which 'All As are Bs' fails to hold, where the given restricted generalization is understood along the proposed simple-addition restricted conditional.

The question to ask is why all As are Bs ought to follow from the corresponding subset claim. The answer, of course, seems obvious: this follows by definition. And so it does. The question, however, concerns the definition in question. And this is where the Ripley objection falters. If our set theory is classical, then we should expect-when talking only about classical sets (say, the ZFC universe) - that all As are Bs is a material (i.e., hook) restricted generalization that detaches in the given 'domain' $\sqrt{2}$ But in this case, there's no reason to think that the target B-vicinity theories cannot enjoy as much ${ }^{13}$ the target generalizations simply aren't the simple-addition generalizations (and needn't be, since the hook suffices in the given context). On the other hand, if our set theory is non-classical, then only details will tell whether Ripley's objection gets off the ground. After all, it may well be that, in the non-classical 'set theory', the simple-addition conditional is used to define 'subset', in which case the model theory might deliver the results that Ripley's objection claims are absent. But, again, only details will tell. Either way, the objection fails to establish a problem with the simple-addition proposal (at least as one among various detachable restricted conditionals).

\subsection{ONE MORE REPLY}

One more reply points to an ambiguity in restricted generalizations. In short, we have two restricted conditionals, one that detaches and one that doesn't. In addition to the simple-addition conditional, which I've proposed for the former role, we have the hook: $\alpha \supset \beta$ or, in primitive notation, $\neg \alpha \vee \beta$. This is not detachable; however, it is true in the sort of models to which Ripley points. On this reply, what the Ripley objection highlights is that neither of the two restricted conditionals (the simple-addition and the hook) is in general adequate for all purposes; however, it may be that for any purpose, one or the other conditional suits. This is for future work to tell.

\section{CLOSING REMARKS}

Relevant logics, and the theories closed under them, have long faced an issue concerning restricted generalizations. A recent discussion of the problem is in [3], wherein one solution is given. This paper reports the good news that, by using 'simple addition', we meet the desiderata laid out in [3], and so achieve a restricted conditional in a simple, fairly theory-neutral fashion. Moreover, and more good news, the chief objection to the proposal (viz., Ripley's objec-

\footnotetext{
${ }^{12} \mathrm{My}$ 'domain' talk is a sloppy way of saying that, in effect, the hook is detachable in the theory ZFC: you won't find $\alpha$ and $\alpha \supset \beta$ in the given theory without finding $\beta$.

${ }^{13}$ In fact, some of them do [2, [O].
} 
tion) may be answered along one of various lines. The task now is to push the proposal further. Such is future work. ${ }^{14}$

\section{REFERENCES}

[r] Jc Beall. Logic: The Basics. Oxford: Routledge, 2010.

[2] Jc Beall. Spandrels of Truth. Oxford: Oxford University Press, 2009.

[3] Jc Beall and Ross Brady and Alan Hazen and Graham Priest and Greg Restall. Relevant Restricted Quantification. Fournal of Philosophical Logic. Vol 35, No. 6, 2006, pp. 587-598.

[4] N. D. Belnap. Restricted quantification and conditional assertion. Truth, Syntax, and Modality. Edited by Hugues Leblanc. Amsterdam: North Holland Publishing Co., I973.

[5] Sam Butchart. Binary Quantifiers for Relevant Paraconsistent Logic. To appear. Presented at the Australasian Association for Philosophy conference in Melbourne, 2008.

[6] Ross Brady. Universal Logic. Stanford, CA: CSLI Lecture Notes, 2006.

[7] J. Michael Dunn and Greg Restall. Relevance Logic. Handbook of Philosophical Logic (Second Edition). Edited by Dov M. Gabbay and Franz Günthner. Dordrecht: D. Reidel, 2002.

[8] Hartry Field. Saving Truth from Paradox. Oxford: Oxford University Press, 2008.

[9] Edwin Mares. Relevant Logic: A Philosophical Interpretation. Cambridge: Cambridge University Press, 2004.

[ro] Graham Priest. In Contradiction (Second Edition). Oxford: Oxford University Press, 2006. First printed by Martinus Nijhoff in 1987.

[II] Graham Priest. An Introduction to Non-Classical Logic (Second Edition). Cambridge: Cambridge University Press, 2008. First edition published in 200I.

[12] Greg Restall. How to be Really Contraction-Free. Studia Logica. Vol. 52, pp. 38I-391, 1993.

[I3] John Slaney. Relevant logic and paraconsistency. Inconsistency Tolerance. Edited by L. Bertossi and A. Hunter and T. Schaub. Heidelberg: Springer-Verlag 2004.

[I4] John Slaney. The implications of paraconsistency. Proceedings of the I2th International foint Conference on Artificial Intelligence, I991.

${ }^{\mathrm{I}}$ I'd like to thank an anonymous referee whose comments not only improved this paper, but also contained very interesting suggestions that, due to schedule pressures, I regrettably could not incorporate. Again, I look forward to future work. 
The Australasian fournal of Logic (ISSN I448-5052) disseminates articles that significantly advance the study of logic, in its mathematical, philosophical or computational guises. The scope of the journal includes all areas of logic, both pure and applied to topics in philosophy, mathematics, computation, linguistics and the other sciences.

Articles appearing in the journal have been carefully and critically refereed under the responsibility of members of the Editorial Board. Only papers judged to be both significant and excellent are accepted for publication.

The journal is freely available at the journal website at

http://www .philosophy . unimelb.edu.au/ajl/

All issues of the journal are archived electronically at the journal website.

SUBSCRIPTIONS Individuals may subscribe to the journal by sending an email, including a full name, an institutional affiliation and an email address to the managing editor at ajl-editors@unimelb.edu.au Subscribers will receive email abstracts of accepted papers to an address of their choice. For institutional subscription, please email the managing editor at ajl-editors@unimelb.edu.au.

Complete published papers may be downloaded at the journal's website at http://www.philosophy.unimelb.edu.au/ajl/ The journal currently publishes in pdf format.

Submission The journal accepts submissions of papers electronically. To submit an article for publication, send the ${ }^{\Delta} \mathrm{T}_{\mathrm{E}} \mathrm{X}$ source of a submission to a member of the editorial board. For a current list of the editorial board, consult the website.

The copyright of each article remains with the author or authors of that article. 